\title{
Rotational dynamics in the plastic-crystal phase of ethanol: Relevance for understanding the dynamics during the structural glass transition
}

\author{
A. Criado \\ Departamento de Física de la Materia Condensada, and Instituto de Ciencia de Materiales de Sevilla, Universidad de Sevilla-CSIC, \\ P.O. Box 1065, E-41080, Seville, Spain \\ M. Jiménez-Ruiz, C. Cabrillo, and F. J. Bermejo \\ Consejo Superior de Investigaciones Científicas, Serrano 123, 28006 Madrid, Spain \\ R. Fernández-Perea \\ Argonne National Laboratory, Argonne, Illinois 60439 \\ H. E. Fischer \\ Institut Laue Langevin, BP 156x, F-38042 Grenoble Cedex 9, France \\ F. R. Trouw \\ Argonne National Laboratory, Argonne, Illinois 60439 \\ (Received 22 July 1999; revised manuscript received 22 October 1999)
}

\begin{abstract}
The reorientational dynamics within the rotationally disordered cubic plastic phase of solid ethanol is investigated by means of the concurrent use of computer molecular dynamics and quasielastic neutron scattering. Motions involving widely different time scales are shown to take place above the calorimetric "glass transition" which is centered at $T_{g} \approx 97 \mathrm{~K}$. These correspond to well-defined reorientations belonging to the cubic point group. The dynamics of this solid exhibits features remarkably close to those of the supercooled liquid that can exist at the same temperature. Such similitude of dynamic behavior serves to provide some clues for the understanding of the nature of molecular motions at temperatures close to the canonical liquid $\rightarrow$ glass transition.
\end{abstract}

\section{INTRODUCTION}

The present paper deals with the reorientational dynamics of plastic-crystalline (PC, rotator-or rotationally disordered-phase) ethanol, a cubic bcc crystal (space group $\operatorname{Im} \overline{3} m),{ }^{1}$ which is the phase showing thermodynamic stability just above that of the deeply supercooled liquid (SCL). Under its own vapor pressure this phase can be stabilized for relatively long periods of time and for temperatures up to $T_{m}^{\mathrm{PC}} \approx 125 \mathrm{~K}$, where it melts previous to a rapid recrystallization into the orientationally ordered monoclinic phase. The formation of the bcc phase involves a partial ordering mechanism leading to the emergence of a long-range-ordered array of the molecular centers of mass, while the macroscopic density and orientational correlations still retain characteristics highly reminiscent of those found for the SCL. ${ }^{1,2}$ This transformation is achieved by means of relatively small molecular rearrangements which have to take place within a highly viscous liquid. It is precisely such huge viscosity $\left(\simeq 10^{10}\right) \quad \mathrm{P}$ that provides the stabilization of this phase against full ordering into the monoclinic crystal $(P c$ space group). This has been recently proven by means of real time neutron diffraction ${ }^{3}$ where a rather large activation energy for crystallization into this phase was found, i.e., $E_{a}^{\mathrm{PC}}$ $=3.2 \mathrm{eV}$.

If expressed in terms of ratios relative to the structural glass-transition temperature $T_{g}=97 \mathrm{~K}$, one finds that the range of existence of the $\mathrm{PC}$ phase extends to $T_{m}^{\mathrm{PC}} / T_{g}$ $=1.29$, while melting of the stable crystal occurs at $T_{m} / T_{g}$ $=1.64$. Below $97 \mathrm{~K}$ molecular movements become increasingly sluggish. ${ }^{4}$ As the temperature is further decreased, leading to a complete arrest of high-amplitude motions below $\approx 80 \mathrm{~K}$, an orientationally disordered state (ODC, glassy-crystal or orientational-glass OG) is attained. Freezing of molecular motions thus takes place within the same range of temperatures encompassing the liquid $\rightarrow$ glass transition and manifests itself as a calorimetric phase change involving the freezing of $\approx 2.8$ degrees of freedom, ${ }^{4}$ as well as a moderate jump in the lattice coefficient of thermal expansion.

Apart from the intrinsic interest in pursuing studies on the dynamics of this, partially ordered system, the main thrust behind the present endeavor lies in the astoundingly close proximity of the dynamic features of the PC phase with those exhibited by the SCL. ${ }^{5}$ In fact, most spectroscopic probes such as dielectric, ${ }^{5}$ light scattering, ${ }^{6,7}$ and neutron scattering ${ }^{6,4,8}$ show a very close parallelism between the dynamic behavior of the SCL and the plastic crystal as well as between the glass and the orientationally disordered phase. In consequence, our hopes are to identify some of the dynamic entities giving rise to a relaxation as proven by the employed spectroscopies, by examining in detail the dynamic response of the PC. Since relaxation within such crystals mostly arises from rotational motions (plus a rotationtranslation coupling of smaller magnitude), and these occur on a crystal lattice, it should then be possible to quantify 
both the characteristic time scales as well as the geometric details of such reorientational excursions.

For these reasons, we will follow procedures involving the concurrent use of neutron spectroscopy and moleculardynamics computer simulations. The use of both handles is required due to the complex dynamics of the system under study. To put this quantitatively, it should suffice to consider that, in general, ${ }^{9}$ molecular reorientations about the axis of a cubic lattice involve five classes of rotations, each one having an associated characteristic (relaxation) time and a structure factor. Under such circumstances, to develop a dynamical model from information supplied only by experiment seems a hopeless task. Thus, our use of the simulations is to facilitate access to details hardly amenable through direct experimental means. As we shall see below, the comparison between simulation and experimental data serves to assign the observed relaxations to microscopic reorientations.

\section{EXPERIMENT DETAILS}

The measurements reported here are somewhat complementary to those already described involving macroscopic scales as explored by dielectric relaxation ${ }^{5}$ and Brillouin light scattering. ${ }^{6,7}$ They concern measurements carried out using instruments with frequency windows about the meV (to explore motions of the order of a picosecond) and $\mu \mathrm{eV}$ (motions within the nanosecond scale). The instrument chosen to explore the former was the quasielastic neutron spectrometer (QENS) inverted-geometry inelastic scattering spectrometer hosted by the IPNS (Intense Pulsed Neutron Source), whereas the latter concern measurements carried on the $\mu \mathrm{eV}$-resolution, backscattering machine IN16 of the Institut Laue Langevin, Grenoble (France). The achieved instrumental resolution at QENS was of the order of $90 \mu \mathrm{eV}$ whereas that of IN16 was of about 1 and $0.8 \mu \mathrm{eV}$ for two different spectrometer settings, having energy-transfer ranges up to \pm 16 and $\pm 6 \mu \mathrm{eV}$, respectively. Sample preparation and the general conditions of the experiment have been described a number of times ${ }^{5,4,8}$ and basically involves annealing the deeply supercooled liquid until the strong (100) Bragg reflections of the bcc structure fully develop and no hint of the liquid main peak centered at $Q \approx 1.7 \AA^{-1}$ remains in diffractograms collected at intervals of a few minutes. Fully deuterated $\left(\mathrm{CD}_{3} \mathrm{CD}_{2} \mathrm{OD}\right)$ and partially deuterated $\left(\mathrm{CD}_{3} \mathrm{CD}_{2} \mathrm{OH}\right)$ samples have been employed in the measurements. Although the presence of a sizeable coherent cross section complicates the analysis in terms of the simplest (incoherent) approximation, the use of partially deuterated samples is dictated by the need of monitoring the state of the sample during annealing, which is done by direct inspection of the diffraction pattern.

Measurements carried out at QENS (involving full- and partially deuterated samples) and IN16 (partially deuterated sample) revealed the presence of quasinelastic broadening of the order of $0.3 \mathrm{meV}$ and $4 \mu \mathrm{eV}$, respectively, as can be gauged from spectra shown in Fig. 1. Spectra at a temperature below (left frame) and above (right frame) the glass transition for each instrument is shown in Fig. 1. A comparison of spectra on both columns shows that the increase in temperature leads to a concomitant increase in both quasielastic intensity and broadening.
A first assessment of the temperature dependence of the measured spectra was attempted by an evaluation of its effective linewidth, which is given in terms of the reduced second and fourth frequency moments ${ }^{10}$ as

$$
\Gamma_{Q}=\frac{\pi}{2 \sqrt{3}}\left[\frac{\left(\bar{\omega}^{2}\right)^{3}}{\bar{\omega}^{4}}\right]^{1 / 2}, \quad \bar{\omega}^{n}=\int d \omega \omega^{n} I(Q, \omega) .
$$

The procedure of common usage in studies of paramagnetic scattering involves the use of ratios between frequency moments of Lorentzian functions. The indefinite integral defining the moments is to be taken first integrating until a cutoff frequency $\omega_{c}$ and taking the limit $\omega_{c} \rightarrow \infty$ afterwards. Such moments are obviously divergent for a Lorentzian. However, the divergencies are linear and cubic in $\omega_{c}$ for the second and fourth moments, respectively, something which ensures that the above-mentioned ratio becomes a well-defined quantity that coincides with $\Gamma_{Q}$ for sufficiently large $\omega_{c}$. A marked dependence of $\Gamma_{Q}$ on temperature was found for both sets of measurements. However, the values for the linewidths estimated in this way were significantly smaller than those estimated by visual inspection or those derived from fits to the observed line shape in terms of simple model consisting of an elastic ( $\delta$-like) plus a Lorentzian component. Such a discrepancy is originated by the slowly decaying tails of the Lorentzian functions which extend well beyond the range of accessible energy transfers. This affects the terms entering Eq. (1) differently and, although it provides correct estimates for the trends with temperature, it needs to be accounted for.

Under the circumstances referred to above, recourse to a relatively simple model scattering law is indicated in order to quantify the temperature dependence of the parameters defining the quasielastic spectra. The obvious choice was a model based on a standard expression for the scattering law for particles reorienting along the cubic crystal axes which reads ${ }^{9}$

$$
\begin{aligned}
I(Q, \omega)= & \exp \left(-\frac{\left\langle u^{2}\right\rangle}{3} Q^{2}\right)\left[A_{0}(Q) \delta(\omega)+S_{\mathrm{rot}}(Q, \omega)\right] \\
& \otimes R(\omega), \\
& S_{\mathrm{rot}}(Q, \omega)=\sum_{J=2}^{5} A_{j}(Q) \frac{1}{\pi} \frac{\tau_{j}}{1+\omega^{2} \tau_{j}^{2}},
\end{aligned}
$$

where $A_{j}(Q)$ are quasielastic structure factors for a particle reorienting under the effects of rotations of the crystal point group, the exponential stands for the Debye-Waller term with mean-square-displacement $\left\langle u^{2}\right\rangle$ (taken from previous determinations of the spectral frequency distribution), $R(\omega)$ is the instrumental resolution, $\delta(\omega)$ is the elastic term with intensity $A_{0}(Q)$, and $\tau_{j}$ are the inverse of the observed quasielastic linewidths.

Both sets of parameters, $\tau_{j}$ 's and $A_{j}(Q)$ 's are temperature dependent and, on the other hand, a minimum of four characteristic relaxation times $\tau_{j}$ is required on symmetry grounds as we shall see below. This makes the analysis of the spectral shapes in terms of fits of the intensities to Eq. (2) an ill-conditioned problem unless additional information (or constraints) are brought forward. 

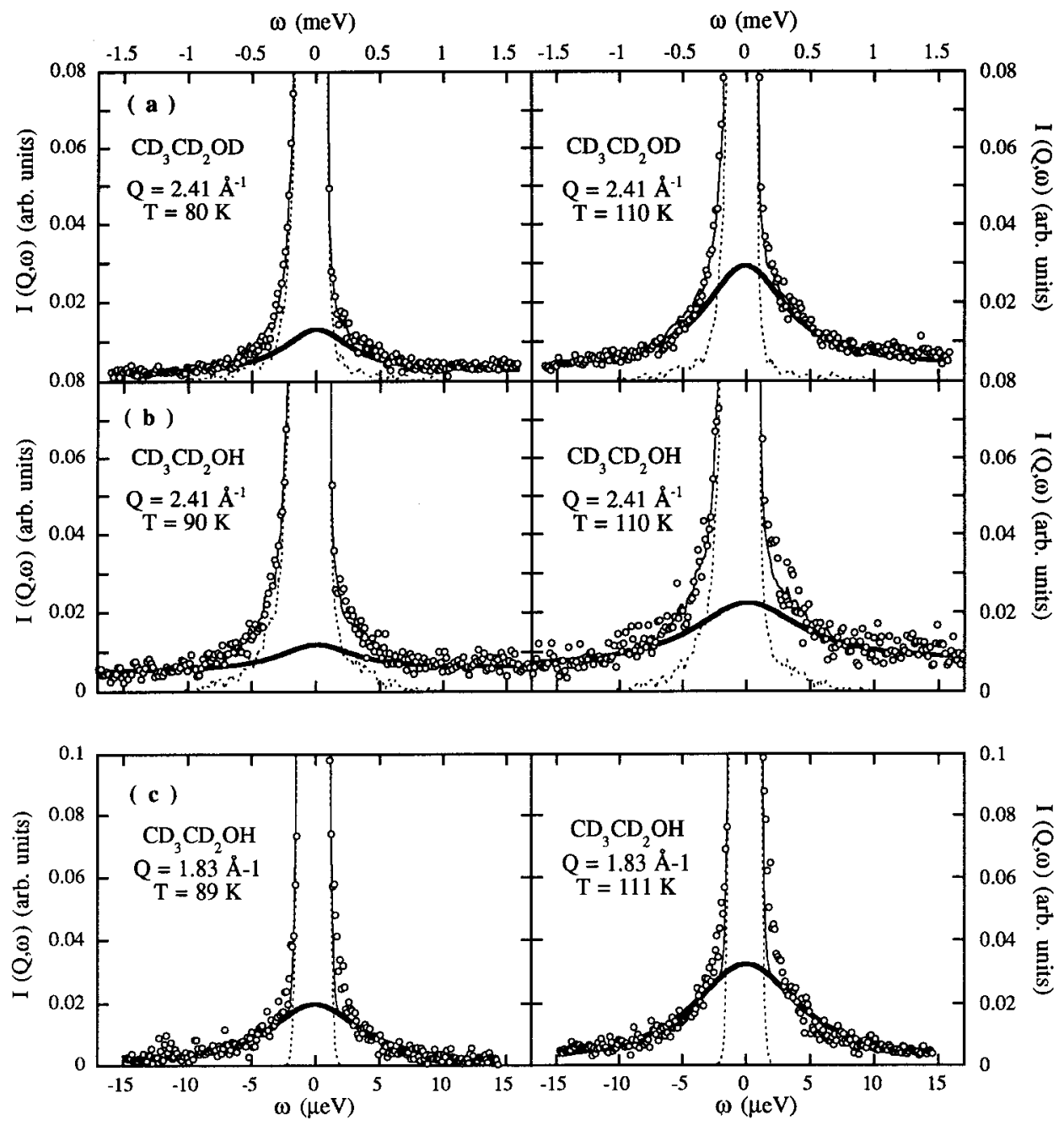

FIG. 1. The upper frames show a spectrum as measured using the QENS spectrometer showing its decomposition into elastic (dashes) and quasielastic (thick solid) components. The lower frame depicts results from IN16. The same symbols are used. The temperature and wave vector are given as insets.

In the present case, additional information is provided from the results of the computer simulations. These predict that the four relaxation times are well separated, and on such a basis one expects that movements within the two very different frequency windows explored by experiment will correspond to just one kind of motion. Under this assumption the measured spectra were modeled in terms of an elastic, $\delta$-like peak plus a quasielastic component (convoluted with the instrument resolution). Finally, it was found that the set of data measured at both scales ( $\mu \mathrm{eV}$ and $\mathrm{meV})$ are affected by the finite range of achievable energy transfers, especially at the higher temperatures where the Lorentzian tails have not fully decayed. This would result in an underestimation of the linewidth which would become more severe as the temperature is raised. To remedy this, the experimental line shapes were fitted introducing a constraint that accounts for the finite value of the maximum energy transfers. The formal procedure is described in some detail in the Appendix. The line-shape analysis was then carried out using Eq. (2), which now ensures that the estimated values for the linewidths are free from truncation effects. The different contributions to the spectra are shown in Fig. 1.

\section{MOLECULAR-DYNAMICS SIMULATION}

Molecular-dynamics (MD) simulations have been successfully employed to study the dynamics of the reorientational motions in plastic crystals. ${ }^{11,12}$ These highly anharmonic systems render inadequate the use of standard lattice dynamical calculations and making it necessary to resort to more realistic methods. Through the trajectories calculated in a MD simulation we can "see" the reorientations or molecular jumps between symmetry-related orientations. In addition one gains access to quantities such as the dynamic structure factors which as shown below are an invaluable tool for the assignment of experimental observations as well as to quantities not accessible experimentally such as the dynamic structure factors for the molecular centers of mass.

In a previous paper ${ }^{1}$ we initiated a structural study of the bcc cubic phase of ethanol using neutron powder diffraction. Unfortunately, only six Bragg peaks are seen over the diffuse background and, on top of this, the lowest angle (110) peak has a very large intensity if compared with the other five. This hindered us in discriminating between different models for the molecular orientation distribution function on the basis of diffraction data only. In fact, an isotropic orientation 
model could fit the experimental Bragg intensities as well as did other more elaborate models, which account for preferred orientations. Here, we aim to gain access to not only timeaveraged properties but also to get some aid for the analysis of the experimental quasielastic neutron spectra. As known from cases previously studied, ${ }^{9}$ the spectra of such solids is composed of relaxational bands associated with molecular reorientations or jumps between symmetrically equivalent preferred orientations. Previous experience shows that the jump time between orientations is considerably shorter than the residence time in one of these preferred orientations. The nature of the jump processes is known to depend on whether the molecular symmetry coincides with the crystal site symmetry, (e.g., such is the case of $\mathrm{SF}_{6}$ ), or is significantly lower than that (such as occurs in crystalline adamantane). In the former case, molecular reorientations are understood as thermally activated processes involving jumps between equipotential orientations whereas in the latter they are a direct consequence of the need to adapt the molecule to the timeaveraged higher-symmetry crystal environment. A low pointgroup symmetry molecule, such as ethanol in a cubic site environment, thus falls within this second case and is expected to reorient dynamically among the different crystal octants.

In principle, one can obtain the time scales of the jump processes from the analysis of the quasielastic profiles. However, the identification of such broadening with specific microscopic motions to characterize the processes involved is not an easy task. Usually, a calculation of the quasielastic scattering as a function of the $\vec{Q}$ vector of momentum transfers is made by adopting several simplified assumptions and the results are compared with experiment in order to infer the geometry of the molecular jumps. This can be done with a good chance of success for molecules of high symmetry where the number of possible reorientations is small and a model involving a few reorientational times can be written down. $^{9}$

The present case is however significantly more complex. For this low-symmetry molecule a large number of different reorientations is expected. A priori knowledge of the orientational probability distribution is necessary in order to find the preferred molecular orientations and hence to adopt a model for the possible different reorientations between them and, as explained above, very little information could be obtained from Rietveld analysis of the diffraction pattern.

\section{A. Simulation details}

The MD simulations have been carried out assuming rigid molecules which can translate and rotate. Atomic Cartesian coordinates are used as variables and the rigid-body condition is introduced as a constraint in the integration of the equation of motion using the Verlet algorithm with a $0.01 \mathrm{ps}$ timestep. An ideal ethanol molecule has been built up assuming standard bond lengths and tetrahedral bond angles. A complete MD model for ethanol would imply a nonrigid molecule where the dihedral angle involving the $\mathrm{OH}$ group could change thus allowing the dynamical transition between the trans and gauche molecular conformations. This requires a good internal molecular force field which does not seem to be available at present. In our case we are interested in

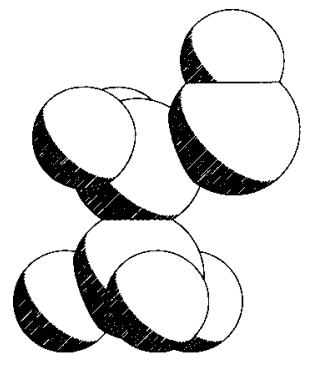

(a)

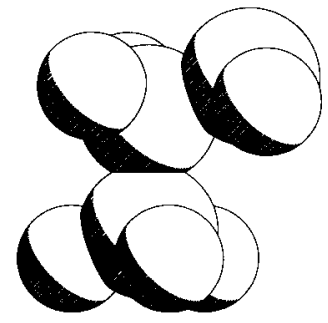

(b)
FIG. 2. Drawing of the ethanol molecule showing the elongated trans conformation and the quasispherical gauche configuration.

whole-molecule reorientational motions at low frequencies and therefore such a primitive model suffices our purposes. To validate the choice of such a primitive model one should recall the evidence from neutron diffraction ${ }^{1}$ pointing to the fact that the proportion of the trans conformation (if any) is small compared to the gauche. In addition, we have tried separate MD runs considering rigid molecules in each conformation. In the trans case a cubic rotator phase could not be stabilized whereas for the gauche conformation a cubic plastic phase is stable with a cubic lattice parameter very close to the experimental one, which confirms the physical soundness of our assumption. From steric arguments it is also evident that the trans molecular conformation has an elongated shape (Fig. 2), which hinders molecular reorientations and makes the formation of a rotator phase difficult, whereas the gauche configuration corresponds to a quasispherical molecular shape typical of other plastic crystals.

Intermolecular forces have been modeled with atom-atom potential functions $V(r)=-A / r^{6}+B \exp (-C r)$, which have been successfully used in crystal packing and latticedynamical studies of molecular crystals. ${ }^{13}$ The parameters entering such a formula are those of Williams and co-workers, ${ }^{14,15}$ which have proven to give very good results in our previous work. ${ }^{16}$ Several different ways to model the electrostatic interactions have been explored. The isolated molecule of ethanol has a dipole moment of $1.71 \mathrm{D},{ }^{17} \mathrm{a}$ value also assumed by several electronic partial charge models used in MD simulations of liquids. ${ }^{17,18}$ Most of these are used in conjunction with models involving at most three force centers. Our attempt to simulate the rotator phase using these point-charge models failed because they seem to grossly overestimate the electrostatic interactions and the plastic phase seems to be very sensitive to this. In fact, such models for the electrostatics induce high nearest-neighbor dipole-dipole orientational correlations which prevent the formation of the rotator phase. On the other hand, screening effects within the real crystal will surely have a strong reduction effect over the point charges with respect to the gasphase values. On the other hand, previous experiments portray the molecular orientations as being fairly isotropic. Moreover, the very nature of a cubic rotator phase in which the molecules rotate very quickly according to the cubic point symmetry is incompatible with the existence of longlasting orientational correlations such as hydrogen bonds, which have a directional character. In addition, it is a wellknown fact that the very fast molecular reorientations in plastic crystals involve large accelerations which can only be 
induced by nearest-neighbor short-range repulsion forces stemming from the $B \exp (-C r)$ potential term.

The considerations given in the above paragraph put some bounds on the magnitude of the dipole moment to be used. Moreover, since the $r^{-6}$ part of the potential reproduces the plastic and glassy phases in the correct temperature ranges and is able to account for the neutron spectra in terms of characteristic relaxation times, which, as we shall see, compare well with experiment, this proves that Coulomb forces have to be here relatively weak. Also, if we scale the pointcharge models referred to above by dividing the charges by a factor of 2 we can obtain a rotator phase in the MD simulation which gives a computed structural and dynamical behavior very similar to that obtained with the simple 6-exp model so that we cannot discriminate between these two models.

The simulation procedure employed here closely follows that described in Ref. 11. A simulation box containing 3 $\times 3 \times 3$ cubic unit cells, i.e., 54 molecules have been found suitable to tackle the cyclic boundary condition effects. The molecules were initially arranged in a bcc Bravais lattice corresponding to the experimental cell parameter. The initial configuration was orientationally ordered. Strong orientational correlations soon disappeared after thermallization to the desired temperature. The latter was started by giving the molecules the random velocities that were attained. The simulations have been carried out in the NPT ensemble ${ }^{19}$ meaning that the unit-cell shape and size are allowed to fluctuate at constant pressure. In this way, we eliminate any externally imposed restriction upon the structure and we can study the stability of the cubic phase as a function of temperature.

\section{B. MD calculation of the time-averaged structure}

The simulated bcc crystal shows a lattice parameter and a range of temperatures within which it is stable, which agree with experiment. The analysis of the molecular trajectories shows that between 95 and $120 \mathrm{~K}$ the orientations are dynamically disordered and the molecules perform quick reorientations on the sites of a bcc Bravais lattice. Below $95 \mathrm{~K}$ an orientational glass $(\mathrm{OG})$ state is progressively attained as a consequence of the molecular kinetic energy not being able to overcome the rotational potential energy barriers. The results of the simulation are again very encouraging since they mimic the behavior of the real crystal. Whereas we cannot observe any clearly measurable difference in the molecular rotational behavior in the 95-120 K temperature range, the analysis of the rotational motion shows that for temperatures below $95 \mathrm{~K}$ the molecules still perform sluggish reorientations and are dynamically disordered but the rate of reorientations decreases considerably reflecting the freezing of the rotational motion. This behavior becomes more pronounced the lower the temperature. At $50 \mathrm{~K}$ the molecules perform reorientations typically every $150 \mathrm{ps}$.

Figure 3 shows the evolution with temperature of the calculated lattice parameter. The experimental value is of $5.369 \AA$ at $90 \mathrm{~K}$ and the difference comes to be about $3 \%$ which is bearable for the kind of atom-atom potentials we are using. The temperature dependence of the lattice constant departs from linearity for temperatures below $50 \mathrm{~K}$. At these

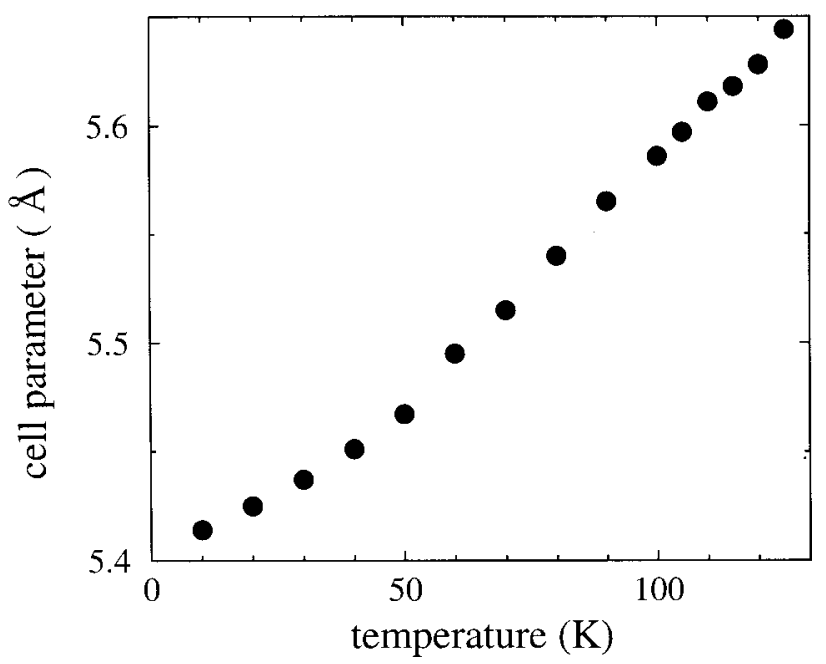

FIG. 3. Temperature dependence of the lattice parameter in the MD simulation for the rotator phase (RP) and the orientational glass (OG) cubic phase of ethanol.

low temperatures the intermolecular distances are shorter and the short-range repulsion forces make the cell contraction more difficult.

\section{RESULTS}

\section{A. Experimental observations}

\section{Dynamics on picosecond scales}

The temperature dependence of the experimental linewidths, as measured using the QENS spectrometer, is shown in Fig. 4 for both samples. Apart from details arising from the different scattering power, the same dependence with temperature is observed, showing that the broadening of the quasielastic spectrum increases through the $\mathrm{OG} \rightarrow \mathrm{PC}$ rotational melting transition.

Apart from the variation with temperature of the linewidth, the $\mathrm{PC} \rightarrow \mathrm{OG}$ transition is also followed by the rise in temperature of the quasielastic intensity and the concomitant decrease of the elastic peak, as is also shown in Fig. 5. As seen there, the transition is very nicely monitored following the transfer of spectral intensity from elastic to quasi- (or

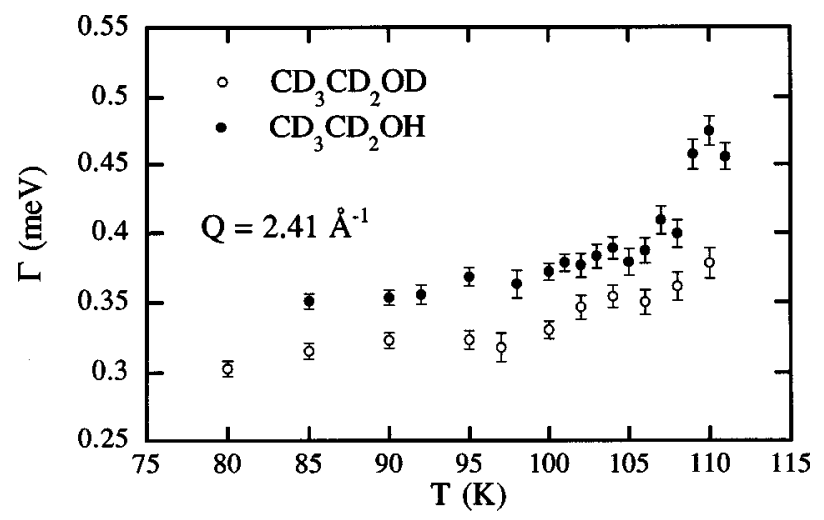

FIG. 4. Temperature dependence of the quasielastic linewidths as measured using QENS for the full and partially deuterated samples. 


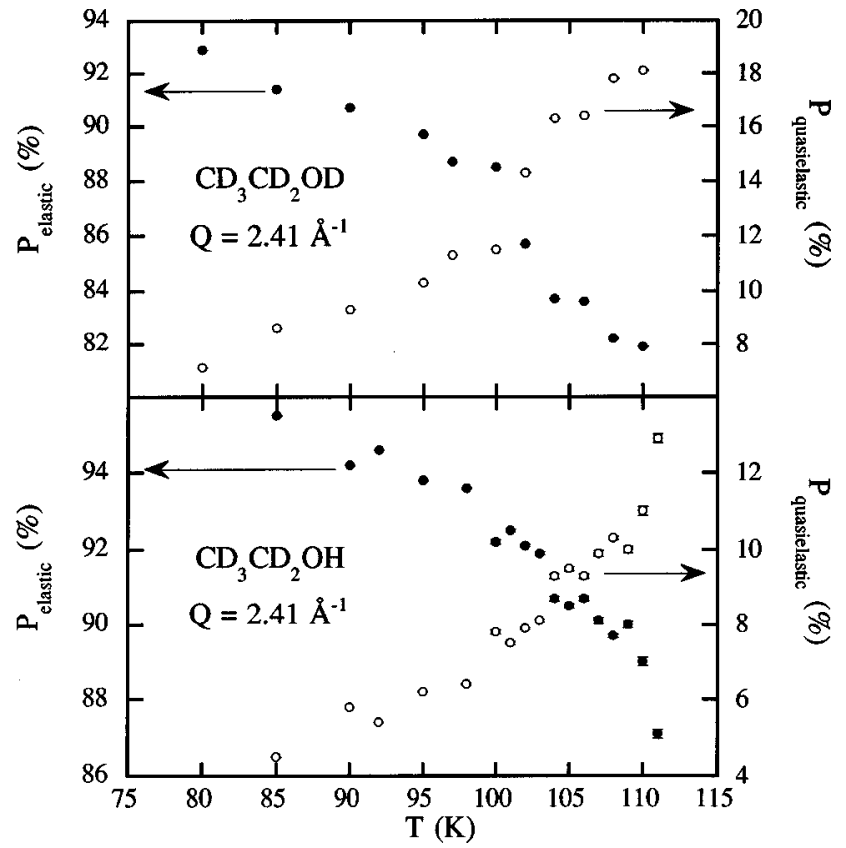

FIG. 5. Temperature dependence of the elastic and quasielastic intensities measured at meV scales. The labels on the ordinate axis refer to the fraction of the elastic (quasielastic) intensity over the total-energy-integrated intensity.

inelastic) scattering. This contrasts with the case of the liquid $\rightarrow$ glass transition, where the spectral parameter most sensitive to the transition is the Debye-Waller term.

From the present data it is seen that molecular rotations occur in the rotator-phase crystal on a scale of about 1 ps. Below the glass transition the rotational freezing seems complete since the quasielastic width only has a slight temperature dependence. The finite value may well arise from the excess of modes characteristic of the glasses. ${ }^{7}$ Well below 85 $\mathrm{K}$ rotational freezing is complete and all the molecular degrees of freedom that are thermally excited will contribute to the spectrum as inelastic (e.g., finite-frequency) signals ${ }^{4}$ only. This is in very good agreement with specific-heat data, ${ }^{4}$ which also shows that the extent in temperatures of the transition is quite comparable to that evidenced by the present data (i.e., about $18 \mathrm{~K}$ ).

\section{Dynamics within the nanosecond scales}

Fully equivalent results to those measured using QENS are shown in Figs. 6 and 7 which were obtained using the IN16 spectrometer. In other words, molecular jumps involving scales within the nanosecond range freeze when the PC $\rightarrow$ OG transition is approached. Apart from a process involving linewidths of the order of $4 \mu \mathrm{eV}$, which is exemplified by data shown in the aforementioned graphs, a second, slower motion with characteristic linewidths of the order of about $1.5 \mu \mathrm{eV}$ was also detected using the higher-resolution instrument configuration (maximal energy transfers of $\pm 6 \mu \mathrm{eV})$, and in fact some indications of the presence of such a slower component are seen in data shown in Fig. 1. The temperature evolution of this second relaxation followed a pattern quite similar to that of the $4 \mu \mathrm{eV}$ broadening, although the measured data covered temperatures up to $102 \mathrm{~K}$ only.

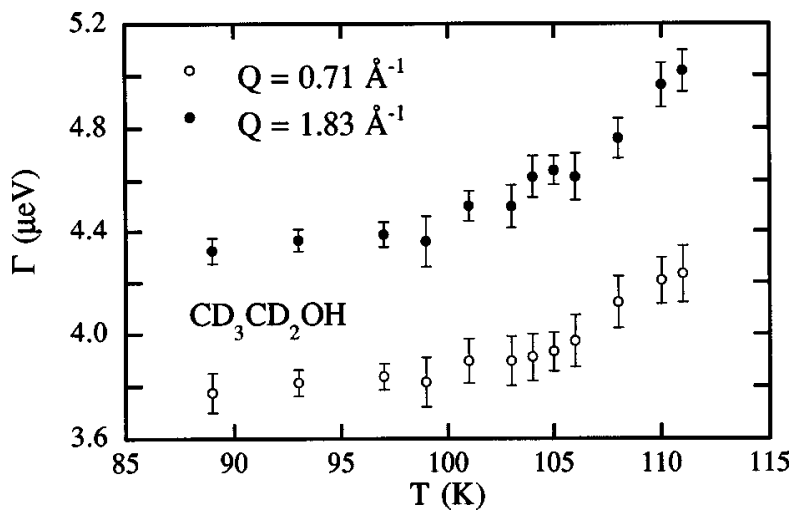

FIG. 6. Temperature dependence of the quasielastic linewidths as measured using IN16.

\section{B. Results from molecular dynamics}

\section{Orientational probability distribution and rotational dynamics}

The usual approach to specify the orientational probability distribution of plastic crystals consists of an expansion given in terms of symmetry-adapted spherical harmonics. The required coefficients are reduced down to a manageable number only when both the site and the molecular symmetry are high since some coefficients become zero. The approach is here impractical for the reasons mentioned above. In this case, the simulated data provide an alternative route since the instantaneous molecular orientations are stored as a function of time. Even so, some difficulties persist since the calculation of the molecular orientation distribution requires the use of the full three Euler angles, which are difficult to handle. A representation in terms of the polar-angle molecular direction distributions makes things easier to interpret. For a lowsymmetry molecule such as ethanol the choice of the relevant molecular directions is not trivial. We have found that the orientational distributions are conveniently described in terms of the polar-angle distributions of the C-O and C-C bonds. Such polar angles, $\theta$ and $\phi$ measured with respect to the crystal axes, have been stored for 800 ps. On the other hand, by recourse to the cubic symmetry of the problem at hand, they are reduced to the range $0 \leqslant \theta \leqslant 90^{\circ}, 0 \leqslant \phi$ $\leqslant 90^{\circ}$.

The orientational distributions are then specified by twodimensional plots where each pair of $(\theta, \phi)$ coordinates is

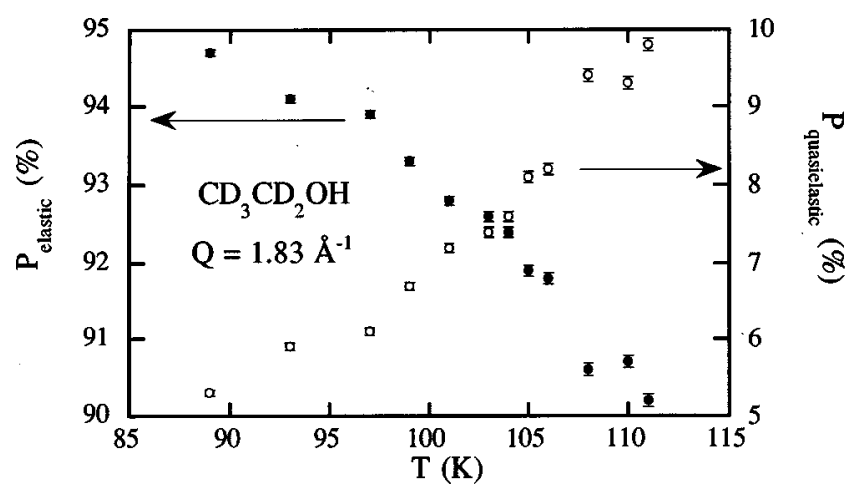

FIG. 7. Temperature dependence of the elastic and quasielastic intensities at $\mu \mathrm{eV}$ scales. The labels on the ordinate axis refer to the fraction of the elastic (quasielastic) intensity over the total energyintegrated intensity. 


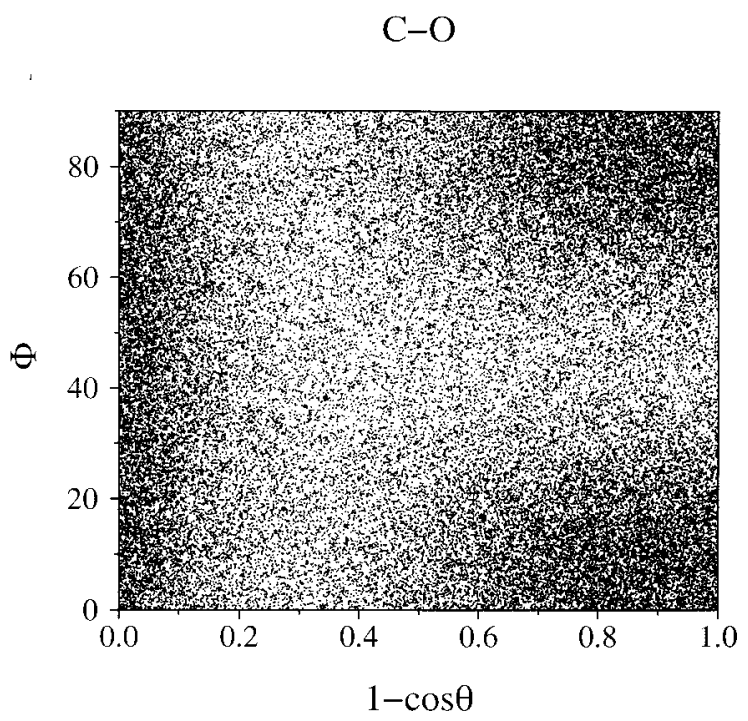

FIG. 8. Polar representation of the orientational distribution of the $\mathrm{C}-\mathrm{O}$ bond with respect to the Cartesian crystal axes reduced to the first octant.

represented as a dot. To make things clearer, we show plots of $\phi$ vs $x=1-\cos \theta$, where $0 \leqslant x \leqslant 1$. This is an isometric spherical representation where an isotropic distribution of orientations would yield a plot with a uniform density of dots. The results for the $\mathrm{C}-\mathrm{O}$ and $\mathrm{C}-\mathrm{C}$ bonds are shown in Figs. 8 and 9, respectively. Both figures reflect the threefold cubic symmetry as seen by the density of points within regions about $(0, \phi),\left(90^{\circ}, 0^{\circ}\right)$, and $\left(90^{\circ}, 90^{\circ}\right)$ which correspond to the three [100] directions. The data shown by both figures enables us to draw a clear picture of the average orientational behavior of a single molecule. There are some preferred orientations, with the $\mathrm{C}-\mathrm{O}$ bonds mostly aligning along the [100] directions whereas the $\mathrm{C}-\mathrm{C}$ bonds do it along the $[111]\left(x=0.42, \phi=90^{\circ}\right)$. This means that the C-O bond preferentially lies on the cube edge and the $\mathrm{C}-\mathrm{C}$ bond lies on the cube diagonal. As all diagonals and edges are equivalent

\section{$\mathrm{C}-\mathrm{C}$}

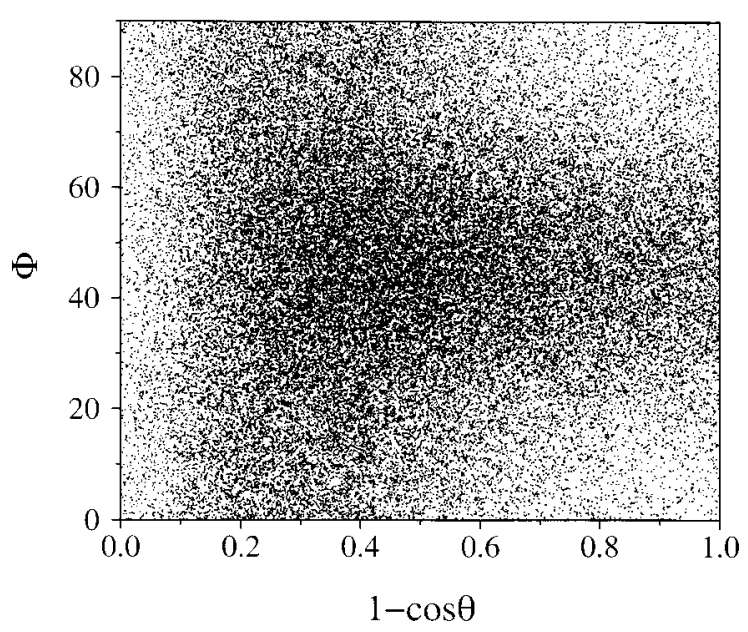

FIG. 9. Polar representation of the orientational distribution of the $\mathrm{C}-\mathrm{C}$ bond with respect to the Cartesian crystal axes reduced to the first octant.
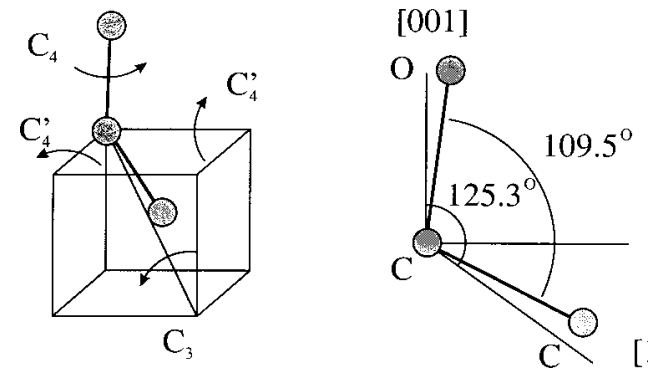

FIG. 10. Drawing showing the preferred orientation of the C-C-O skeleton with respect to the crystal directions together with the crystal and molecular angle geometries. The different kinds of detected molecular reorientations $C_{4}, C_{4}^{\prime}$, and $C_{3}$ are also represented.

by symmetry, the molecule would perform reorientations among the 24 different such basic orientations in order to compensate for the different crystal site and molecular point symmetries. A sketch of the orientation of the molecule relative to the cubic cell is given in Fig. 10. We can see that the ideal C-C-O angle is $109.5^{\circ}$ whereas the angle between the

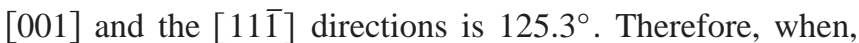
for instance, the $\mathrm{C}-\mathrm{O}$ bond aligns with its maximum probability direction the $\mathrm{C}-\mathrm{C}$ bond departs some $15.8^{\circ}$ from the cube diagonal, and vice versa. Such a departure from the ideal shape is usually compensated by large librational thermal motions about the most probable molecular orientation. To quantify such a deviation we represent in Fig. 11 the C-O probability distribution function as a function of the angular departure from the [100] direction. There we see that the distribution width absorbs this molecular geometry departure from the crystal symmetry directions.

The orientational distribution of the direction normal to the C-C-O plane shows maxima at the [110] directions as might be expected. There is, however, a subtle feature in that the difference between the maximum and minimum dot densities is reduced with respect to the other two distributions indicating that the normal stays less time around the most probable orientation than in the other two cases. This particular detail is useful for understanding how the molecules

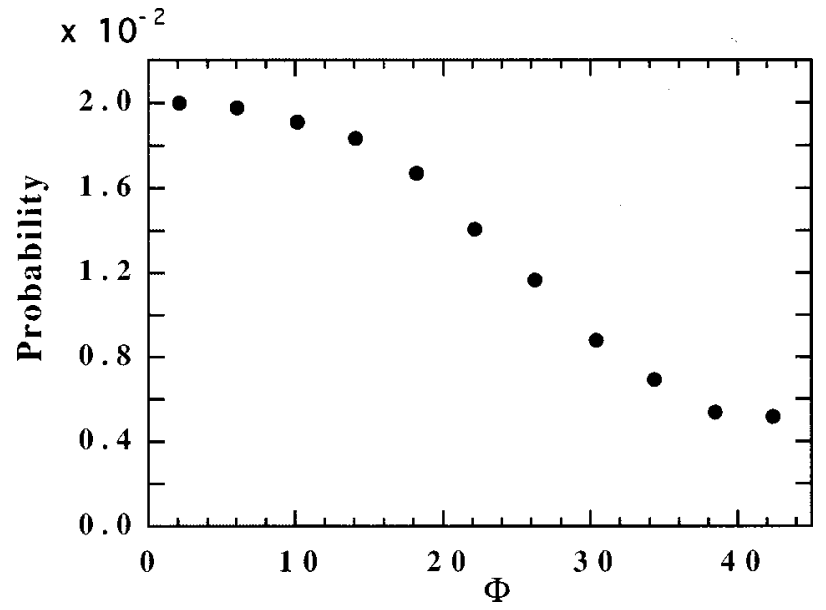

FIG. 11. Cut of the C-O orientational function (Fig. 8) starting at $\left(\theta=90^{\circ}, \phi=0\right)$ and ending at $\left(\theta=90^{\circ}, \phi=45^{\circ}\right)$. The dots in Fig. 8 have been binned at intervals of $4^{\circ}$ when producing this plot. 
perform the jumps between symmetry-related orientations. It has to do with the room left empty by the surrounding molecules so that the central molecule can fit into a new position. Those molecular rotations involving small angles and leaving some atoms in the same position are more likely to occur than those requiring large-angle rotations where all the atoms move to new positions. Therefore, the most probable reorientations will be those bringing the $\mathrm{C}-\mathrm{O}$ bonds to all [100] directions and the $\mathrm{C}-\mathrm{C}$ bonds to all [111] directions. These are $90^{\circ}$ rotations about the $\mathrm{C}-\mathrm{O}$ bonds $\left(C_{4}\right)$ and $120^{\circ}$ rotations about the $\mathrm{C}-\mathrm{C}$ bond $\left(C_{3}\right)$ as it is illustrated in Fig. 10. In either case, one of the bonds is kept invariant whereas in both cases the normal to the $\mathrm{C}-\mathrm{C}-\mathrm{O}$ plane reorients to a new direction. This explains why the maximum of the distribution is less pronounced in this case due to the time spent in the reorientation process. Additional reorientations are also possible and these will correspond to other rotation elements of the cubic symmetry group. These are the fourfold rotations $\left(C_{4}^{\prime}\right)$ about the two [100] directions perpendicular to the $\mathrm{C}-\mathrm{O}$ bond, the threefold rotations $C_{3}^{\prime}$ about the three [111] crystal directions different from that of the $\mathrm{C}-\mathrm{C}$ bond and finally, the twofold rotations $C_{2}$ about the [110] crystal directions. It is worth pointing out the difference between our case and that for an octahedral molecule $\mathrm{SF}_{6}$ for instance), where all $C_{4}, C_{3}$, and $C_{2}$ rotations are equivalent. In our case, due to the molecular low symmetry, each kind contains nonequivalent rotations, each of them possibly having a different jump rate, which is given as the inverse of characteristic relaxation times.

\section{Estimation of the jump rates}

Extracting information on the jump rates from the computed trajectories becomes fairly involved as the picture of molecules occupying a few well-defined equilibrium orientations performing instantaneous reorientations to another position is obviously an oversimplification. Rather, the molecules execute librations about preferred orientations and it is difficult sometimes to decide whether the molecules have jumped or not, and also, the molecules may initiate a jump but they may go back to their previous position (aborted jumps). For these reasons, the analysis of the molecular reorientations is most conveniently carried out by visual means, plotting the molecular orientation as a function of time and identifying the possible jumps. In such a way we have tracked the occurrence of the different reorientational motions. The jump rates estimated following such a procedure should obviously be considered as tentative estimates, given the aforementioned difficulties. The most frequent reorientations are those already referred to, the $C_{4}$ rotation about the $\mathrm{C}-\mathrm{O}$ bond and the $C_{3}$ rotation about the $\mathrm{C}-\mathrm{C}$ bond. At $105 \mathrm{~K}$ these occur with frequencies of $\approx 0.4$ and $0.2 \mathrm{meV}$, respectively. Also, we have detected reorientations $C_{4}^{\prime}$ about [100] directions perpendicular to the $\mathrm{C}-\mathrm{O}$ bond, those having a lower frequency of $70 \mu \mathrm{eV}$. We have been unable to detect either $C_{3}^{\prime}$ or $C_{2}$ rotations during an evolution time of 500 ps for a given molecule. This means that such reorientations have a period of at least several hundreds of picoseconds. Summarizing, we get a picture in which the main reorientational mechanisms are $90^{\circ}$ rotations around the $\mathrm{C}-\mathrm{O}$ bonds and $120^{\circ}$ about the $\mathrm{C}-\mathrm{C}$ bonds although $C_{4}^{\prime}$ reorientations do occur as well albeit with substantially reduced frequencies.

\section{MD calculation of the spectral frequency distributions}

The dynamics of a plastic crystal can be studied from the point of view of the collective excitations or phonons and also studying the so-called single-molecule behavior where the quantities of interest represent the dynamics of the solid as "viewed" by a tagged molecule. We have first carried out a number of calculations of the spectrum of collective excitations along the crystal highest symmetry directions. As expected, the result shows the presence of heavily damped acoustic excitations which become clearly visible for $Q$ values well below the Brillouin-zone boundary only, alongside a number of branches of "optical" character appearing at somewhat higher frequencies. The spectra get even more structureless after a polycrystalline average is taken. Therefore, our main interest is here focused onto single-molecule properties such as the linear and angular autocorrelation functions which can be directly compared with experiment. These are defined as

$$
Z(t)=\frac{\left\langle\vec{v}\left(t_{0}\right) \vec{v}\left(t_{0}+t\right)\right\rangle}{\left\langle\left|\vec{v}\left(t_{0}\right)\right|^{2}\right\rangle}
$$

and

$$
C(t)=\frac{\left\langle\vec{\omega}\left(t_{0}\right) \vec{\omega}\left(t_{0}+t\right)\right\rangle}{\left\langle\left|\vec{\omega}\left(t_{0}\right)\right|^{2}\right\rangle},
$$

where the averages (angular brackets) run over all molecules and all the initial times $t_{0}$. Here, $\vec{v}$ and $\vec{\omega}$ are the linear velocity of the molecular center of mass and the angular velocity of the molecule, respectively. The power spectra of these functions are related to the translational and rotational density of states (DOS). Figure 12 show the direct and Fourier-transformed functions for the translational and rotational motions. From such graphs it is seen that $Z(t)$ and $C(t)$ go through a region of negative values before decaying to zero indicating that the velocities change their sign. Such behaviors which are also characteristic of the dynamics of the liquid state are usually referred to as the "cage effect" or, in other words provide indications of motions taking place within a quasistable configuration of the neighboring particles. This means that the molecules oscillate and also librate, i.e., perform rotational oscillations superimposed to a component of rotational diffusion, characteristic of a rotator phase as can be inferred from the nonzero value of the Fourier transform at $\omega=0$. This behavior is similar to that displayed by $\mathrm{C}_{2} \mathrm{Cl}_{6}$, for instance, ${ }^{11}$ and different from that of the plastic phase of methane,$^{20}$ where the molecules seem to rotate without executing librations. It is also interesting to notice that the translational power spectrum shows linear behavior as $\omega \rightarrow 0$ instead of the expected $Z(\omega) \propto \omega^{2}$ Debye law for the acoustic modes in a harmonic crystal. This also happens in some other plastic crystals and has been traditionally ascribed to the effects of rotation-translation coupling which hinders the existence of well defined (i.e., propagating) acoustic modes in the crystal. 


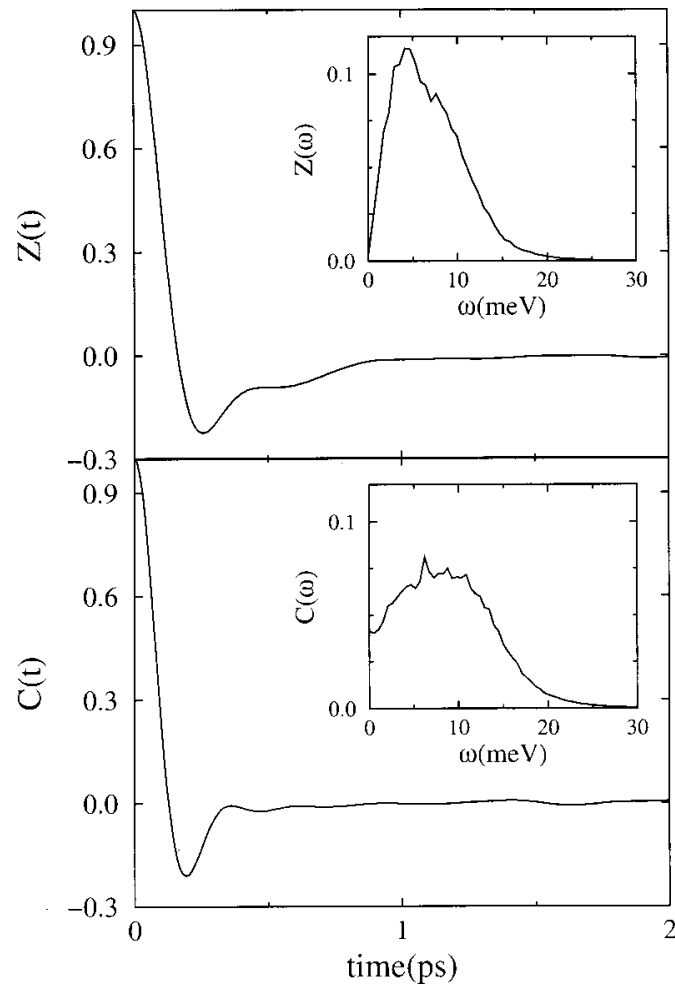

FIG. 12. The upper frame shows the center-of-mass velocity autocorrelation function $Z(t)$ and its Fourier transform $Z(\omega)$ which is shown in the inset. The lower frame depicts the calculated angular velocity autocorrelation function $C(t)$ and its Fourier transform $C(\omega)$ (shown in the inset).

The calculated quantities can readily be compared with experimental data measured at $105 \mathrm{~K}$. The latter are shown in Fig. 13, which in turn provides a comparison of the lowfrequency data for the plastic and orientationally ordered (monoclinic) crystals. The latter, apart from showing a more structured shape, approaches $\omega=0$ following a Debye law,

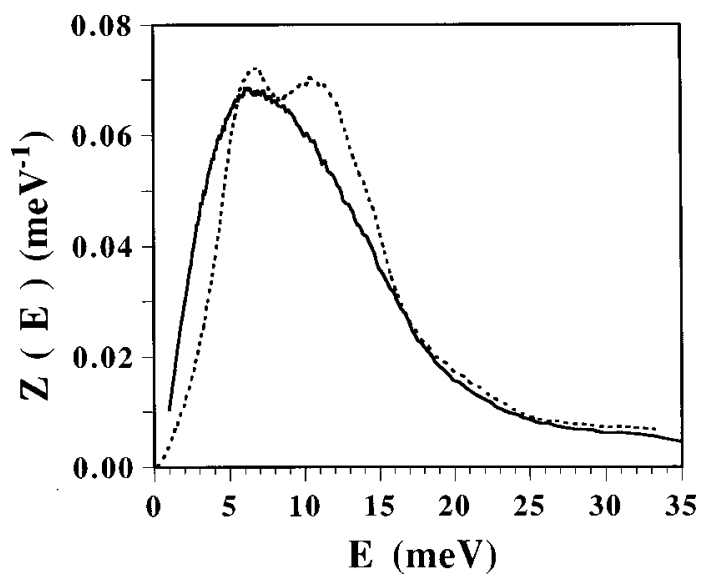

FIG. 13. Experimental estimate for the frequency distribution of the plastic crystal as measured at $105 \mathrm{~K}$ on a full hydrogenous sample (solid line) (Ref. 21). The dashed line depicts the spectrum for the ordered monoclinic crystal measured at the same temperature. Both spectra have been derived by means of procedures described in Ref. 22. Data for the plastic crystal at low frequencies would require the use of an extrapolation procedure similar to those employed for liquids [see, for instance, (Ref. 26)].
TABLE I. Experimental $F_{o b s}$ (Ref. 1) and calculated $F_{c a l}$ Bragg intensities using Eq. (7).

\begin{tabular}{ccc}
\hline \hline$(h k l)$ & $F_{\text {obs }}$ & $F_{\text {cal }}$ \\
\hline$(110)$ & $31.8(2)$ & 30.9 \\
$(200)$ & $7.5(4)$ & 10.5 \\
$(211)$ & $5.6(5)$ & 8.0 \\
$(220)$ & $2.5(7)$ & 7.5 \\
$(310)$ & $4.2(5)$ & 3.5 \\
$(222)$ & $1.9(9)$ & 5.1 \\
\hline \hline
\end{tabular}

while the rotator-phase crystal shows a large deviation from this in much the same way as commented above for the simulated spectra.

From a comparison of the graphs shown in Figs. 12 and 13 it is seen that both the maxima and the extent in frequency of experimental and calculated functions are in good agreement. Notice that the high-frequency tail of the experimental function contains contributions from the internal molecular dynamics which cannot obviously be accounted for in the rigid-body model used for the calculation. Even so, both experiment and calculation show maxima at about $6 \mathrm{meV}$ and most of the spectral power is exhausted for frequencies higher than $20 \mathrm{meV}$. In other words, the high-frequency dynamics of the rotator-phase crystal seems to be well accounted for by the relatively simple model used to represent the intermolecular interactions in the MD simulation.

\section{Calculation of the elastic and inelastic structure factors}

To shed some additional light into the static structure of the rotator-phase crystal and thus overcome some of the difficulties referred to above ${ }^{1}$ which arose as a consequence of the reduced number of Bragg peaks seen in diffraction experiments, we have calculated the the coherent elastic structure factor. It is derived from the coherent density operator and is defined as the Fourier transform of the instantaneous scattering length of the crystal:

$$
\rho(\vec{Q}, t)=\sum_{i} b_{i} \exp [i \vec{Q} \vec{r}(i, t)]
$$

where $\vec{Q}$ is the neutron momentum transfer and $b_{i}$ and $\vec{r}(i, t)$ are the coherent scattering length and the instantaneous position of atom $i$. The elastic Bragg scattering arises from the time average of the density operator:

$$
I_{B}=|\langle\rho(\vec{Q}, t)\rangle|^{2} .
$$

Using this expression, the calculation of the Bragg intensity has been carried out for time averages of 500 ps and the results are shown in Table I together with the experimental intensities extracted from the powder-diffraction profile. As can be inferred from the table the Bragg intensity pattern is reasonably well described by experiment. Higher-order reflections are somewhat overestimated in the calculation because of the use of a rigid molecule model. This is so because the internal molecular vibrations do play a role in reducing the intensities of these high-angle reflections by means of increasing the exponent entering the Debye-Waller terms. As regards the previous exercise carried out consider- 
ing diffraction data only, ${ }^{1}$ the present results show that a model of finite-angle jumps between symmetry-allowed orientations (i.e., a Frenkel model ${ }^{1}$ ) is better suited for describing the nature of molecular rotations.

We have also calculated the single-particle dynamic structure factor which is to be compared with that measured by incoherent inelastic neutron scattering in order to study the single-molecule rotational dynamics. The spectra were calculated to help with the analysis of experimental data. In the case of incoherent scattering, the total scattering intensity is the sum of the intensities scattered by every atom in the crystal. For a given $\vec{Q}$ we can define an instantaneous incoherent atomic density operator as

$$
\rho_{i}^{s}(\vec{Q}, t)=b_{i}^{s} \exp [i \vec{Q} \vec{r}(i, t)],
$$

where $i$ labels the different atoms in the crystal and $b^{s}$ is the incoherent scattering length $\left(\sigma_{\text {inc }}=2 \pi b^{s 2}\right)$. An incoherent intermediate autocorrelation function can be calculated as

$$
F^{s}(\vec{Q}, t)=\sum_{i}\left\langle\rho_{i}^{s}(\vec{Q}, 0) \rho_{i}^{s *}(\vec{Q}, t)\right\rangle,
$$

such that the incoherent inelastic spectra would be its Fourier time transform. Here, $\langle\cdots\rangle$ means time average over all instantaneous configurations along the MD runs of typically $1000 \mathrm{ps}$ in our case.

The $F^{s}(\vec{Q}, t)$ functions decay with time to a constant value which is equal to the incoherent elastic contribution arising from the time-averaged scattering terms:

$$
I_{\text {elas }}^{s}(\vec{Q})=\sum_{i}\left|\left\langle\rho_{i}^{s}(\vec{Q}, t)\right\rangle\right|^{2}
$$

It is usually convenient to subtract this term from $F^{s}(\vec{Q}, t)$ in order to get a function which decays to zero for long times as it is in the coherent case, also avoiding truncation errors when calculating its time Fourier transform.

In this way, we have calculated the intermediate autocorrelation function for different values of the scattering vector. Long runs of $1000 \mathrm{ps}$ have been used in order to achieve convergence in the estimation of the elastic incoherent term. In spite of this, some curves still show a decay to a constant value which differs slightly from zero. Whether this result is fully significant and underpins some long-time feature characteristic of glassy-dynamics (i.e., a two-step relaxation) could not be ascertained. At any rate, such a feature does not significantly affect the calculation of the characteristic relaxation times obtained from an exponential fit of the curves.

The most significant result from the calculated $F^{s}(\vec{Q}, t)$ concerns their strong, nonexponential shape. A set of such functions is displayed in Fig. 14 for several values of momentum-transfer $Q$. A minimum of four exponential decays are required to fit the shapes of the calculated curves, all of them having well separated decay constants. In fact the curves show a first short-time decay arising from collective (phonon) modes having characteristic times of the order of hundredths of a picosecond or lower, a second decay of a few tenths of a ps, and two additional relaxations with times of a few and close to $10 \mathrm{ps}$. In other words, the observed

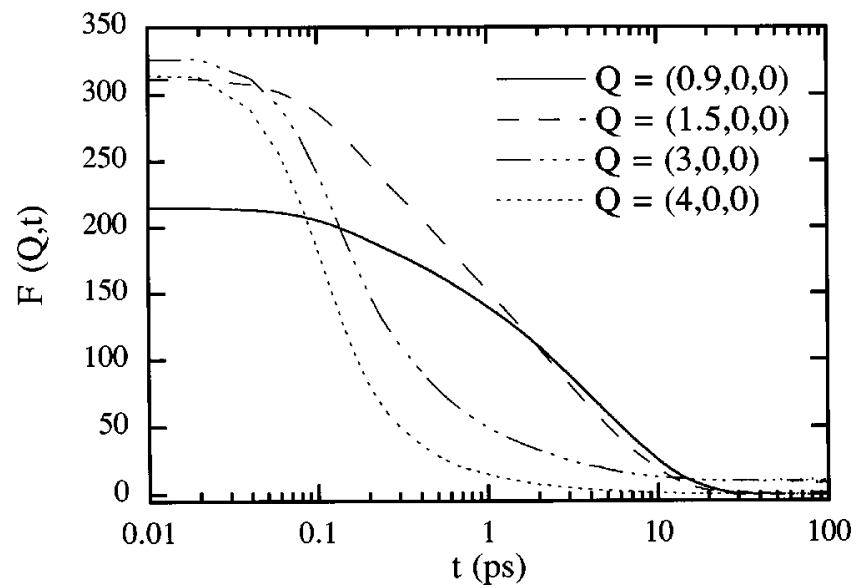

FIG. 14. A set of calculated $F^{s}(Q, t)$ curves for momentum transfers given as insets.

deviation from ideal behavior arises from the widely different time scales found for different kinds of reorientations.

\section{DISCUSSION AND CONCLUSIONS}

The main aim of the present study has been the identification of the microscopic entities causing relaxations at scales of pico- and nanoseconds within the rotator-(plastic) phase of ethanol. The material has been recently used as a model for investigating the glassy dynamics and, in view of the proximity of dynamic behavior of the plastic- and supercooled liquid, it is expected that some of the results should be of relevance for studies on glassy matter.

From data and arguments described in the preceeding section a tentative assignment of the broadenings detected in the quasielastic neutron-scattering experiments to molecular reorientations can be made. Motions taking place within the $\mathrm{meV}$ (picosecond) scale nicely correspond to those $C_{4}$ rotations about the $\mathrm{C}-\mathrm{O}$ bond and the $C_{3}$ rotations around the $\mathrm{C}-\mathrm{C}$ bond found in the MD simulations. Such reorientations should involve excursions of angles as large as $90^{\circ}$ and $120^{\circ}$ about the referred axes. On the other hand, the quasielastic signals observed within the $\mu \mathrm{eV}$ scale do have to be identified with those $C_{3}^{\prime}$ and $C_{2}$ motions also referred to in the previous paragraphs.

As mentioned above, while experiment shows that both the quasielastic linewidth and the spectral intensities increase with temperature, only an increase in the intensity of the quasielastic spectrum is clearly observed in the simulations for temperatures above $95 \mathrm{~K}$. Rotational freezing below this temperature as observed experimentally is accounted for by the calculation, although it appears as a process significantly more sluggish than that actually observed. Whether the quasielastic linewidth of the simulated spectra increases with temperature or not could not be ascertained. The main difficulty lies in the fact that, as shown by experiment, one would need to achieve very high statistical accuracy to mimic the variations in linewidth actually measured.

The complicated picture that arises from the MD study, allows one to infer the reasons why the wave-vector dependence of the quasielastic structure factors depart substantially from that corresponding to simple models of molecular reorientation. ${ }^{9}$ Such a complex pattern of reorientations gives 
rise to strongly nonexponential relaxations as a direct consequence of the disparate time scales involved. The result seems to be of relevance regarding present debates on "dynamic heterogeneity" 23 of relaxation patterns in disordered matter since, as proven by a number of experimental tools the reorientational dynamics of the SCL is expected to share the most significant features with those exhibited by the plastic crystal.

The rotational freezing transition studied here involves the sluggish arrest of about 3 (2.8) degrees of freedom, as seen by calorimetric means. Such a transition is of a purely dynamic character and in fact, its main features are retained by a conceptually simple model of hard needles reorienting in a bcc lattice. ${ }^{24} \mathrm{~A}$ detailed comparison between the parameters used to monitor the rotational freezing ${ }^{24}$ shows that molecular rotations ocurring at vastly different time scales arrest within a very narrow temperature interval. The implications for the canonical liquid $\rightarrow$ glass transition are clear since the latter certainly involves a large reorientational component. ${ }^{5}$ Furthermore, such an analogy is also expected on the basis of the remarkably close proximity of the local structures of the SCL and rotator phase crystal. ${ }^{1}$ Such similitude leads one to expect that the torques experienced by individual molecules should be fully comparable in both phases and therefore the individual reorientations which come as a consequence of such forces should share common characteristics. This has been ascertained by dielectric experiments ${ }^{5}$ which show that the similitude in behavior is even closer for relaxations with frequencies beyond the main $(\alpha)$ peak. In fact, features on the leading high-frequency edge of such a peak (the "wing" or "shoulder" characteristic of both PC and SCL) as well as higher-frequency $(\beta)$ relaxations, show dependences with temperature describable by basically the same parameters.

A further point worth investigating concerns the possible role of molecular rotations such as those described in the present work as the entities responsible for restoring ergodicity at temperatures above the glass transition $T_{g}$ but below that $T_{c}$ where motions are predicted to be arrested by theories of the mode-coupling family (MCT) ${ }^{25}$ As shown here, individual molecular reorientations can still take place under huge viscosities since the SCL or PC retain a substantial free volume in which rotations can take place. Such a mechanism should, therefore, be in operation on most of the materials employed as benchmarks for tests of the MCT predictions and therefore can account in part for the observed deviations.

The comparison between the calculated and measured spectral distributions has shown that the relatively simple model used to model the interparticle potential in the MD study retains the most significant features up to frequencies well within the microscopic realm (above some $20 \mathrm{meV}$ ). Also, both calculation and experiment show some features commonly interpreted as fingerprints of "glassy dynamics." These are a substantial deviation of the frequency distribution $Z(\omega)$ over the Debye law, i.e., the so-called "excess modes," the appearance of a negative region in the velocity autocorrelation functions ("cage effect") and the strong damping of longitudinal-acoustic excitations because of the translation-rotation coupling.

As a main conclusion, the present work shows that relaxations taking place within a model for the deeply super- cooled liquid can be assigned to precise microscopic motions. The results may help to shed some light onto some topics currently under discussion on matters concerning the dynamics of glasses as well as the glass transition.

\section{ACKNOWLEDGMENTS}

This work was supported in part by Grant No. DGICYTPB95-0072-C03 (Spain). Work at ANL was supported by the U.S. Department of Energy, BES-Materials Sciences, under Contract No. W-31-109-ENG-38.

\section{APPENDIX}

From Eq. (2) the spectrum for a given value of the momentum transfer $Q$ is modeled assuming that the instrumental resolution is given by a normalized Gaussian with standard deviation $\sigma$ and the experimental frequency window allows us to resolve only one of the four Lorentzians. The expression then reads

$$
\begin{aligned}
S(Q, \omega)= & \left(\frac{I_{q e l}}{\pi} \frac{\Gamma}{\omega^{2}+\Gamma^{2}}+I_{e l} \delta(\omega)\right) \\
& \otimes \frac{1}{\sqrt{2 \pi \sigma^{2}}} \exp \left(-\frac{\omega^{2}}{2 \sigma^{2}}\right) .
\end{aligned}
$$

It is given in terms of a quasielastic line strength $I_{q e l}$, a Lorentzian of half width at half maximum linewidth $\Gamma$ and a component representing the elastic intensity $I_{e l}$. The values for the relevant parameters are derived by fits to the measured intensities, while $\sigma$ is estimated from the measured instrument response. The symbol $\otimes$ stands for the convolution operation.

Let $\omega_{c}$ be the cutoff frequency imposed by the experimental setup. Such a cutoff frequency may be considered as infinite if compared with the Gaussian resolution function, but has to be taken care of because of the slow decaying wings of the quasielastic signal.

Under such conditions, the first three even frequency moments of $S(Q, \omega)$ as given by Eq. (A1) are

$$
\begin{gathered}
I_{t o t}=k \Gamma \exp \left(-\frac{\Gamma^{2} \omega^{2}}{2 \sigma^{2}}\right) \otimes \arctan \left(\tilde{\omega}_{c}\right)+I_{e l}, \\
\bar{\omega}^{2}=k \Gamma^{3} \exp \left(-\frac{\Gamma^{2} \omega^{2}}{2 \sigma^{2}}\right) \otimes\left[\tilde{\omega}_{c}-\arctan \left(\tilde{\omega}_{c}\right)\right]+I_{\text {elas }} \sigma^{2}, \\
\bar{\omega}^{4}=k \Gamma^{5} \exp \left(-\frac{\Gamma^{2} \omega^{2}}{2 \sigma^{2}}\right) \otimes\left[\frac{\tilde{\omega}_{c}^{3}}{3}-\tilde{\omega}_{c}+\arctan \left(\tilde{\omega}_{c}\right)\right]+3 I_{e l} \sigma^{4},
\end{gathered}
$$

where $k=\sqrt{2} I_{q e l} /(\sigma \pi \sqrt{\pi})$ and $\tilde{\omega}_{c}=\omega_{c} / \Gamma$. The convolutions appearing in these expressions should be understood as

$$
\exp \left(-\frac{\Gamma^{2} \omega^{2}}{2 \sigma^{2}}\right) \otimes f\left(\tilde{\omega}_{c}\right)=\int_{\infty}^{\infty} d \omega \exp \left(-\frac{\Gamma^{2} \omega^{2}}{2 \sigma^{2}}\right) f\left(\tilde{\omega}_{c}-\omega\right) .
$$


Since the convolution products involving $\arctan \left(\tilde{\omega}_{c}\right)$ cannot be done analytically, we have chosen to eliminate the convolution with $\arctan \left(\tilde{\omega}_{c}\right)$ between the zero and the second moments and the convolution with $\tilde{\omega}_{c}-\arctan \left(\tilde{\omega}_{c}\right)$ between the second and the fourth moments. Once the remaining convolutions are calculated we obtain

$$
\begin{gathered}
\bar{\omega}^{2}=\Gamma^{2}\left(I_{\text {elas }}-I_{\text {tot }}\right)+\Gamma \frac{2 I_{q e l}}{\pi} \omega_{c}+I_{\text {elas }} \sigma^{2}, \\
\bar{\omega}^{4}=\Gamma^{2}\left(I_{\text {elas }} \sigma^{2}-\bar{\omega}^{2}\right)+\Gamma \frac{2 I_{q e l}}{3 \pi}\left(\omega_{c}^{3}+3 \sigma^{2} \omega_{c}\right)+3 I_{\text {el }} \sigma^{4} .
\end{gathered}
$$

Finally, eliminating terms linear in $\Gamma$ in the above equations leads to

$$
\begin{aligned}
& \Gamma^{2}\left[\left(I_{\text {tot }}-I_{\text {elas }} \sigma\right)\left(\omega_{c}^{2}+3 \sigma^{2}\right)-3\left(\bar{\omega}^{2}-I_{\text {elas }} \sigma^{2}\right)\right] \\
& \quad=3\left(\bar{\omega}^{4}-I_{\text {elas }} \sigma^{4}\right),
\end{aligned}
$$

so that

$$
\Gamma=\sqrt{\frac{3\left(\bar{\omega}^{4}-I_{\text {elas }} \sigma^{4}\right)-\left(\bar{\omega}^{2}-I_{\text {elas }} \sigma^{2}\right)\left(\omega_{c}^{2}+3 \sigma^{2}\right)}{\left(I_{\text {tot }}-I_{\text {elas }}\right)\left(\omega_{c}^{2}+3 \sigma^{2}\right)-3\left(\bar{\omega}^{2}-I_{\text {elas }} \sigma^{2}\right)}} .
$$

The procedure we have followed thus involves calculating the moments using the measured spectrum under the constraint given by the above expression. This was found to improve the stability of the fitting problem to a very significant extent.
${ }^{1}$ F.J. Bermejo, A. Criado, R. Fayos, R. Fernández-Perea, H.E. Fischer, E. Suard, A. Guelylah, and J. Zúñiga, Phys. Rev. B 56, 11 536 (1997).

${ }^{2}$ R. Fayos, F.J. Bermejo, J. Davidowski, H.E. Fischer, and M.A. Gonzalez, Phys. Rev. Lett. 77, 3823 (1996).

${ }^{3}$ M. Jiménez-Ruiz, Ph.D. Thesis, Universidad Autónoma de Madrid, 1999.

${ }^{4}$ C. Talón, M.A. Ramos, S. Vieira, G.J. Cuello, F.J. Bermejo, A. Criado, M.L. Senent, S.M. Bennington, H.E. Fischer, and H. Schober, Phys. Rev. B 58, 745 (1998).

${ }^{5}$ M.A. Miller, M. Jiménez-Ruiz, F.J. Bermejo, and N.O. Birge, Phys. Rev. B 57, R13 977 (1998); M. Jiménez-Ruiz, M.A. González, F.J. Bermejo, M.A. Miller, N.O. Birge, I. Cendoya, and A. Alegría, ibid. 59, 9155 (1999).

${ }^{6}$ A. Criado et al., Phys. Rev. B 61, 8778 (2000).

${ }^{7}$ H.E. Fischer, F.J. Bermejo, G.J. Cuello, M.T. Fernandez-Díaz, J. Davidowski, M.A. González, H. Schober, and M.A. JiménezRuiz, Phys. Rev. Lett. 82, 1193 (1999).

${ }^{8}$ M.A. Ramos, S. Vieira, F.J. Bermejo, J. Davidowski, H.E. Fischer, H. Schober, M.A. González, C.K. Loong, and D.L. Price, Phys. Rev. Lett. 78, 82 (1997).

${ }^{9}$ M. Bee, Quasielastic Neutron Scattering (Adam Hilger, Bristol, 1988), p. 222.

${ }^{10}$ S.W. Lovesey, Theory on Neutron Scattering from Condensed Matter (Oxford Science, New York, 1984), p. 326.

${ }^{11}$ A. Criado and A. Muñoz, Mol. Phys. 83, 815 (1994); F. Affouard and M. Descamps, Phys. Rev. B 59, R9011 (1999).

${ }^{12}$ A. Muñoz and A. Criado, Mol. Phys. 84, 1207 (1995).

${ }^{13}$ A.J. Pertshin and A.I. Kitaigorodsky, The Atom-Atom Potential Method (Springer-Verlag, Berlin, 1987).

${ }^{14}$ D.E. Williams, J. Chem. Phys. 47, 4680 (1967).

${ }^{15}$ S.R. Cox, D.E. Williams, and L. Hsu, Acta Crystallogr., Sect. A: Cryst. Phys., Diffr., Theor. Gen. Crystallogr. 37, 293 (1981).

${ }^{16}$ F.J. Bermejo, A. Criado, A. de Andrés, E. Enciso, and H. Schober, Phys. Rev. B 53, 5259 (1996).

${ }^{17}$ M.A. González, E. Enciso, F.J. Bermejo, and M. Bée, J. Chem. Phys. 110, 8045 (1999).

${ }^{18}$ W.I. Jorgensen, J. Phys. Chem. 90, 1276 (1986).

${ }^{19}$ M. Parrinello and A. Rahman, Phys. Rev. Lett. 45, 1196 (1980).

${ }^{20}$ D.G. Bounds, M.L. Klein, and G.N. Patey, J. Chem. Phys. 72, 5348 (1980).

${ }^{21}$ F.J. Bermejo et al., in Complex Behavior of Glassy Systems, edited by M. Rubí et al., Springer Lecture Notes in Physics Vol. 492 (Springer, Berlin, 1997), p. 44.

${ }^{22}$ J. Davidowski, F.J. Bermejo, and J.R. Granada, Phys. Rev. B 58, 706 (1998).

${ }^{23}$ R. Böhmer, Curr. Opin. Solid State Mater. Sci. 3, 378 (1998).

${ }^{24}$ M. Jiménez-Ruiz et al., Phys. Rev. Lett. 83, 2757 (1999).

${ }^{25}$ W. Götze, in Liquids Freezing and the Glass Transition, edited by J.P. Hansen et al. (North-Holland, Amsterdam, 1991), p. 287.

${ }^{26}$ M. Mukherjee et al., Phys. Rev. B 57, R11 031 (1998). 